\title{
Fetal exposure to a maternal low-protein diet is associated with altered left ventricular pressure response to ischaemia-reperfusion injury
}

\author{
Matthew J. Elmes ${ }^{1}$, David S. Gardner ${ }^{2}$ and Simon C. Langley-Evans ${ }^{1}$ \\ ${ }^{1}$ Division of Nutritional Sciences and \\ ${ }^{2}$ School of Veterinary Medicine and Science, University of Nottingham, Sutton Bonington Campus, Loughborough, Leicestershire \\ LE12 5RD, UK
}

(Received 16 October 2006 - Revised 18 December 2006 - Accepted 10 January 2007)

\begin{abstract}
Rats exposed to protein restriction as fetuses develop hypertension as adults. Hypertension increases the risk of myocardial ischaemia and infarction. We investigated whether rats exposed to low-protein diets in utero are more susceptible to myocardial ischaemia-reperfusion (IR) injury. Pregnant Wistar rats were fed control or low-protein (MLP) diets throughout pregnancy. At 4 and 8 weeks postnatal age systolic blood pressure was determined in the offspring using tail-cuff plethysmography. At 6 months of age, rats were treated with saline or $N$-acetylcysteine (NAC) for $48 \mathrm{~h}$. Rapidly excised hearts were retro-perfused (Langendorff) to assess isolated cardiac function before (baseline), during $30 \mathrm{~min}$ ischaemia (no coronary perfusion) and for $60 \mathrm{~min}$ after reinstating coronary perfusion (reperfusion). Hearts were then harvested and treated appropriately for analysis of infarct size. Exposure to the MLP diet in utero significantly increased systolic blood pressure at 4 and 8 weeks of age (6$13 \mathrm{mmHg}$ increase; $P<0.001)$ and significantly impaired recovery of left ventricular developed pressure after ischaemia at 6 months of age in male offspring only $(P<0.003)$. Pre-treatment with NAC prevented this impairment of recovery in MLP male offspring and improved recovery in all females. Myocardial infarct size was not different between dietary groups after IR, but NAC pre-treatment significantly reduced the degree of infarction $(P<0.001)$. In conclusion, an MLP diet throughout gestation significantly impairs recovery of the 6-month-old adult rat heart to IRinduced injury in a sex-specific manner. Undernutrition during development may increase susceptibility to CHD by impairing recovery from coronary events.
\end{abstract}

Fetal programming: Heart: Ischaemia-reperfusion

CVD has traditionally been linked to a poor adult 'lifestyle', for example, a diet high in salt and/or saturated fat, light to moderate smoking and being overweight. Recent epidemiological studies, however, have shown that being underweight and thin at birth increases the risk of developing hypertension (Law et al. 1993) and CVD (Fall et al. 1995) in middle-aged adults. These findings led to the 'fetal programming hypothesis', which suggests that the risk of adult non-communicable disease is partially determined by the environment of the developing fetus (Barker, 1995). Current evidence now suggests that interactions between genetic factors, fetal factors and postnatal diet and 'lifestyle' contribute significantly to the overall risk of developing CVD (Langley-Evans, 2006).

Since our original study showing an association between maternal protein intake and offspring blood pressure (Langley \& Jackson, 1994), a multitude of animal models have been developed to test the fetal programming hypothesis, and these tend to support the idea that early-life nutrition is important in determining the risk of CVD in later life (for a review, see Langley-Evans, 2006). The most widely reported and best-characterised animal model of fetal programming to date is the feeding of low-protein diets during pregnancy in the rat (Langley \& Jackson, 1994; Langley-Evans et al. 1994, 1996a, 1999).

Using this model (Langley \& Jackson, 1994) and others (Woods, 2004), it has been consistently shown that exposure to moderate maternal protein deficiency ( $9 \%$ by weight) leads to low-normal birth weight in rats and other species, and elevates risk factors for cardiovascular and metabolic disease, for example, increased systolic blood pressure, from weaning (about 4 weeks of age) into adult life (Langley \& Jackson, 1994; Langley-Evans et al. 1994, 1996a, 1999). Prenatally programmed hypertension in the rat does not seem to be sex specific, and the timing of protein restriction during pregnancy does not appear critical, as feeding low-protein diets for 1 week only (of a 3-week gestation period) is sufficient to raise blood pressure in the offspring. The magnitude of change in offspring blood pressure is, however, greater when the low-protein diet is targeted to the final week of gestation only (Langley-Evans et al. 1996b; Kwong et al. 2000).

In addition to hypertension, adverse fetal environments lead to structural and functional changes in the neonatal heart. Clinical studies have shown that human fetuses that undergo intra-uterine growth retardation develop left

Abbreviations: CON, control diet; IR, ischaemia-reperfusion; LVDP, left ventricular developed pressure; MLP, maternal low-protein diet; NAC, $N$-acetylcysteine. * Corresponding author: Dr Matthew Elmes, fax +44 115951 6122, email matthew.elmes@ nottingham.ac.uk 
ventricular hypertrophy (Veille et al. 1993; Leipala et al. 2003). Prenatal protein restriction during sheep pregnancy increases both weight of the fetal heart and cardiac expression of key genes involved in cardiac remodelling (Han et al. 2004). In contrast, rats exposed to low-protein diets in utero have a decreased heart weight and cardiomyocyte number in newborn rat pups (Corstius et al. 2005). Fetal hypoxia also has adverse effects on the adult rat heart; it increases its susceptibility to myocardial ischaemia-reperfusion (IR) injury (Li et al. 2003; Xu et al. 2006).

The mechanisms that drive myocardial IR-induced cardiac injury are, as yet, unresolved, but evidence suggests that an early burst of reactive oxygen species upon reperfusion may play a key role (Bolli et al. 1989; Kaplan et al. 1997). Previous studies have shown that in a model of prolonged cardiac ischaemia, antioxidant treatment before (Williams et al. 1991), or after (Ambrosio et al. 1987) the challenge improves postischaemic ventricular function. $N$-acetylcysteine (NAC) is a potent free radical scavenger (Aruoma et al. 1989) and treatment with NAC has been shown to significantly reduce cardiac damage after experimentally induced cardiac IR injury (Sochman et al. 1990; Alberola et al. 1991).

To date, no study has shown that prenatal undernutrition alone can programme a predisposition toward myocardial IR injury. We first hypothesised that exposure to a proteinrestricted diet in utero increases the susceptibility and extent of myocardial injury in the adult rat heart following IR. Second, we hypothesised that prior treatment with the free radical scavenger NAC would blunt the degree of IR injury experienced. We used the reproducible isolated heart preparation (Langendorff) to evaluate ex vivo whole organ function in prenatally protein-restricted offspring and induced $30 \mathrm{~min}$ ischaemia to determine susceptibility of adult rat hearts to IR injury. Further groups either did or did not receive treatment with NAC before the Langendorff procedure, to establish whether the mechanism underpinning nutritionally induced susceptibility to IR injury involved increased or decreased availability of intrinsic coronary reactive oxygen species. Since hypertension and IHD are as prevalent in women as they are in men (Peterson \& Raynor, 2002) the study additionally aimed to examine whether there are sex-specific risk factors for susceptibility to IR injury.

\section{Materials and methods}

\section{Animals}

The present investigation was performed in accordance with the Home Office Guidance on the Operation of the Animals (Scientific Procedures) Act 1986 (Great Britain Home Office, 2000). Sixteen virgin Wistar rat dams (Harlan Ltd, Belton, Leics, UK) aged 10 weeks or older weighing between 250 and $300 \mathrm{~g}$ were mated in the University of Nottingham animal facility. Once mating was confirmed by the appearance of a vaginal plug on the cage floor, the rats were allocated to be fed either a control diet $(\mathrm{CON} ; 180 \mathrm{~g}$ casein $/ \mathrm{kg}$ ) or a lowprotein diet (maternal low-protein diet; MLP; $90 \mathrm{~g}$ casein $/ \mathrm{kg}$ ) as described previously in detail (Langley \& Jackson, 1994). The pregnant rats were then fed the isoenergetic semi-synthetic diet until giving birth at $22 \mathrm{~d}$ gestation. All rats were then transferred to a standard laboratory chow diet (B\&K
Universal Ltd, Hull, UK) and each litter was culled to a maximum of eight pups (four males and four females) to minimise variation in the pups' nutrition during suckling. In the absence of a cross-fostering design, variation in the postnatal nutrition experienced by the pups cannot be excluded. However, we have previously shown that the protein content of milk produced by both groups of dams was similar (Bellinger et al. 2004).

\section{Determination of non-invasive blood pressure}

Systolic blood pressure and pulse rates were determined in all offspring at both 4 and 8 weeks of age using indirect tail-cuff plethysmography, using an IITC model 229 blood pressure monitor (IITC Life Science, Woodland Hills, CA, USA), as previously reported (Langley-Evans et al. 1996b). To give reproducible blood pressure readings during restraint each rat was acclimatised to a temperature of $27^{\circ} \mathrm{C}$ for $2 \mathrm{~h}$ before blood pressure measurements, which were performed within a period of 2-10 min. This method has been extensively characterised and validated by ourselves and elsewhere (LangleyEvans et al. 1996b; Sherman \& Langley-Evans, 2000). Considerable care is required in the use of this technique. Although tail-cuff plethysmography has been the basis for almost all studies of nutritional programming, it is prone to artifacts associated with restraint stress.

\section{The isolated heart (Langendorff) preparation}

At 6 months of age one male and one female from each litter were randomly selected and anaesthetised using $3 \%$ isofluorane in 2 litres $\mathrm{O}_{2} / \mathrm{min}$ and killed by cervical dislocation. The experiment included animals that had blood pressure determined at the earlier stage. Within $90 \mathrm{~s}$, the heart was excised and cannulated via the aorta to Langendorff perfusion apparatus (AD Instruments, Oxford, UK) and perfused with Krebs-Henseleit buffer (118 mM-NaCl, 4.7 mM-KCl, $1.2 \mathrm{~mm}-$ $\mathrm{KH}_{2} \mathrm{PO}_{4}, 1.2 \mathrm{mM}-\mathrm{MgSO}_{4}, 25 \mathrm{~mm}-\mathrm{NaHCO}_{3}, 11 \mathrm{~mm}$-glucose

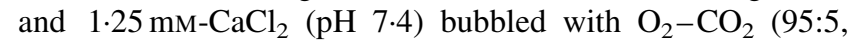
$\mathrm{v} / \mathrm{v}$ ) in a coronary retrograde fashion. Perfusion was maintained at a constant pressure of $60 \mathrm{mmHg}$, with perfusate warmed to $37.4^{\circ} \mathrm{C}$, and the heart immersed in a water-jacketed temperature-controlled glass chamber set at $37.4^{\circ} \mathrm{C}$, therefore ensuring normothermia throughout the perfusion protocol. Contractile function was monitored by the careful insertion of a saline-filled latex balloon into the left ventricle which was adjusted to an end diastolic pressure of between 5 and $10 \mathrm{mmHg}$. Left ventricular and perfusion pressure were continuously monitored through pre-calibrated physiological pressure transducers (Senso-Nor 844; AD Instruments). Data were collected and processed using the Powerlab Data Acquisition System (AD Instruments). Data recording was not started until all variables were stable $(15-30 \mathrm{~min})$ with the following $30 \mathrm{~min}$ period considered baseline. Isolated cardiac ischaemia was then rapidly induced by switching off the coronary perfusion apparatus for a 30 min period. After reinstating coronary perfusion at the previous baseline rate, recording continued for a further $60 \mathrm{~min}$ period to assess cardiac responses to reperfusion. At the end of the IR protocol the left ventricle was harvested to determine the extent of myocardial infarction, as described later. 


\section{Determination of a role for $\mathrm{N}$-acetylcysteine in reducing myocardial ischaemia-reperfusion injury}

In order to assess the contribution of intrinsic myocardial oxidative processes to the development of IR injury, one male and one female rat from each CON and MLP litter were randomly selected to be treated twice daily with an intraperitoneal injection of either saline or NAC $(50 \mathrm{mg} / \mathrm{kg}$ ) (Sigma, St Louis, MO, USA) for $48 \mathrm{~h}$ as reported previously (Langley \& Kelly, 1992). Rats were then euthanised and hearts subjected to the Langendorff procedure as described earlier. At the end of the protocol hearts were harvested as described later.

\section{Determination of myocardial infarct size}

At the end of reperfusion the left ventricle was collected and incubated with 2,3,5 triphenyl tetrazolium chloride solution $(1 \%)$ for $20 \mathrm{~min}$ at $37^{\circ} \mathrm{C}$, and then stored in $10 \%$ formalin solution until analysis. Surviving tissue is stained deep red and infarcted tissue a yellow tan colour. Hearts were then frozen and cut into $30 \mu \mathrm{m}$ longitudinal slices using a cryostat, with every twentieth slice being mounted onto a glass microscope slide for analysis. Three randomised digital images of each section were photographed at $\times 40$ magnification with a Nikon TE33 microscope (Tokyo, Japan) fitted with a micro image video camera (Micropublisher 3.3; QImaging, Burnaby, $\mathrm{BC}, \mathrm{Canada})$. Images were analysed for total area, total area of infarct tissue and percentage infarct in the left ventricle as a whole using Image Pro Plus v. 5.0 (Media Cybernetics, Corporation, Silver Spring, MD, USA). In total, 1560 (CON) and 1980 (MLP) images from 1180 sections were analysed to determine myocardial infarct.

\section{Statistical analysis}

All results are presented as mean values with their standard errors with $P \leq 0.05$ considered significant. Systolic blood pressure as determined by tail-cuff plethsmography is influenced by weight, therefore these data were analysed using a mixed linear model allowing for between- and within-litter effects adjusted for weight (Festing, 2006). IR data were split into three distinct groups, $30 \mathrm{~min}$ baseline, recovery 1 (0-30 min post-ischaemia) and recovery 2 (31-60 min postischaemia). The effects of maternal dietary and/or NAC treatment on left ventricular developed pressure (LVDP) before and after IR were analysed using two-way repeatedmeasures ANOVA (SPSS version 9.0; SPSS Inc., Chicago, IL, USA) with sex as a fixed effect. As only one animal from each litter was included in each treatment group, no adjustment for the litter of origin was necessary. Myocardial infarct size was analysed by two-way ANOVA. Where individual differences were indicated, post hoc tests were carried out using the least squared difference.

\section{Results}

Systolic blood pressure and pulse rate

At 4 weeks of age systolic blood pressure was significantly higher in both male and female offspring exposed to the MLP in utero compared with the CON (Table 1). This elevation in systolic blood pressure was not sex dependent and persisted to the second measurement at 8 weeks of age. A significant diet-related elevation in blood pressure (adjusted for body weight) was observed in the MLP group (adjusted blood pressures at 4 weeks of age: CON 117 (SE 2) $\mathrm{mmHg}$, MLP 127 (SE 2) $\mathrm{mmHg} ; P<0 \cdot 001$, for males and females pooled). At 4 weeks of age heart rate was similar between dietary groups (Table 1), but at 8 weeks was significantly increased in MLP offspring (CON 392 (SE 6) beats per min; MLP 423 (SE 7) beats per min; $P<0 \cdot 001$, for males and females pooled). Although blood pressure was not determined beyond 8 weeks of age, our previous studies (Langley-Evans \& Jackson, 1995) have shown that, once established, hypertension persists to at least 10 months of age.

\section{Cardiac function}

Baseline data. Baseline LVDP function of hearts from CONand MLP-fed offspring is shown in Table 2. In saline-treated rats during baseline, there was no effect of sex or dietary treatment on baseline LVDP. Pre-treatment for $2 \mathrm{~d}$ with NAC had no overall effect on baseline LVDP; however, there were significant effects of sex $(P=0 \cdot 04$; females in general had a lower LVDP than males) and a diet $\times \operatorname{sex} \times$ treatment interaction $(P<0 \cdot 01 ;$ CON females during NAC treatment only had a significantly lower baseline LVDP).

Ischaemia-reperfusion data. Ischaemia of the isolated rat heart for $30 \mathrm{~min}$ significantly reduced LVDP function

Table 1. Systolic blood pressure (SBP) and heart rate (HR)

(Mean values with their standard errors; $n 11-31$ )

\begin{tabular}{|c|c|c|c|c|c|c|c|c|c|}
\hline \multirow[b]{3}{*}{ Sex } & \multirow[b]{3}{*}{ Diet } & \multicolumn{4}{|c|}{4 weeks } & \multicolumn{4}{|c|}{8 weeks } \\
\hline & & \multicolumn{2}{|c|}{$\mathrm{SBP}(\mathrm{mmHg})$} & \multicolumn{2}{|c|}{ HR (beats per min) } & \multicolumn{2}{|c|}{$\mathrm{SBP}(\mathrm{mmHg})$} & \multicolumn{2}{|c|}{ HR (beats per min) } \\
\hline & & Mean & SE & Mean & $\mathrm{SE}$ & Mean & SE & Mean & SE \\
\hline \multirow[t]{2}{*}{ Male } & CON & $114 \cdot 4$ & $2 \cdot 4$ & $408 \cdot 1$ & $6 \cdot 4$ & $129 \cdot 0$ & 4.0 & $379 \cdot 2$ & $7 \cdot 4$ \\
\hline & MLP & $127 \cdot 1^{*}$ & 2.4 & 397.9 & $7 \cdot 2$ & $155 \cdot 4^{\star}$ & 3.8 & $417 \cdot 1^{\star}$ & 7.7 \\
\hline \multirow[t]{2}{*}{ Female } & CON & 119.5 & $2 \cdot 8$ & 392.5 & 6.9 & $122 \cdot 1$ & 3.5 & $404 \cdot 2$ & 9.7 \\
\hline & MLP & $125 \cdot 9^{*}$ & 2.9 & 390.0 & 8.2 & $140 \cdot 4^{*}$ & 3.5 & $429.0^{*}$ & $10 \cdot 1$ \\
\hline
\end{tabular}

CON, control diet; MLP, maternal low-protein diet.

${ }^{*}$ Mean value was significantly different from that of the CON animals of the same $\operatorname{sex}(P<0.001)$. 
Table 2. Contractile function (left ventricular developed pressure; LVDP) of the left ventricle before and after ischaemia-reperfusion* (Mean values with their standard errors)

\begin{tabular}{|c|c|c|c|c|c|c|c|c|c|c|c|c|}
\hline \multirow{2}{*}{$\begin{array}{l}\text { Maternal } \\
\text { group }\end{array}$} & \multirow[b]{2}{*}{ Treatment } & \multirow[b]{2}{*}{ Sex } & \multicolumn{2}{|c|}{$\begin{array}{l}\text { Baseline LVDP } \\
\quad(\mathrm{mmHg})\end{array}$} & \multicolumn{2}{|c|}{$\begin{array}{l}\text { LVDP recovery } 1 \\
\qquad(\mathrm{mmHg})\end{array}$} & \multicolumn{2}{|c|}{$\begin{array}{l}\text { LVDP recovery } 2 \\
\quad(\mathrm{mmHg})\end{array}$} & \multicolumn{2}{|c|}{ Recovery 1 (\%) } & \multicolumn{2}{|c|}{ Recovery 2 (\%) } \\
\hline & & & Mean & $\mathrm{SE}$ & Mean & SE & Mean & $\mathrm{SE}$ & Mean & $\mathrm{SE}$ & Mean & SE \\
\hline \multirow[t]{2}{*}{ CON } & Saline & Male & $27 \cdot 4$ & $4 \cdot 1$ & $12 \cdot 02$ & $2 \cdot 3$ & $15 \cdot 4$ & 3.0 & 43.9 & $5 \cdot 2$ & 60.5 & $12 \cdot 6$ \\
\hline & & Female & 30.4 & 3.5 & $9 \cdot 78$ & $1 \cdot 1$ & $10 \cdot 6$ & $2 \cdot 3$ & 35.4 & 6.0 & 39.4 & 9.4 \\
\hline \multirow[t]{2}{*}{ MLP } & Saline & Male & 34.6 & 3.9 & 8.69 & $2 \cdot 3$ & 11.7 & $2 \cdot 7$ & $25.9 \dagger$ & $6 \cdot 3$ & $34.0 \neq$ & $7 \cdot 6$ \\
\hline & & Female & 23.9 & 4.0 & 7.67 & $2 \cdot 7$ & 9.9 & 2.5 & 29.0 & 4.3 & 37.7 & $9 \cdot 0$ \\
\hline \multirow{2}{*}{ CON } & NAC & Male & 41.3 & $7 \cdot 2$ & 11.9 & $4 \cdot 13$ & $13 \cdot 27$ & 4.8 & $32 \cdot 11$ & 9.9 & 39.45 & 17.4 \\
\hline & & Female & 23.9 & 4.0 & 13.71 & 3.5 & $15 \cdot 1$ & 3.0 & 58.9 & 13.4 & $66 \cdot 1$ & 11.7 \\
\hline \multirow[t]{2}{*}{ MLP } & NAC & Male & $32 \cdot 6$ & 3.5 & $12 \cdot 19$ & $2 \cdot 1$ & 15.69 & 3.1 & $46 \cdot 1$ & 12.5 & 54.81 & $13 \cdot 3$ \\
\hline & & Female & 33.0 & 1.5 & 14.09 & $2 \cdot 3$ & 16.43 & $2 \cdot 9$ & $42 \cdot 6$ & 6.5 & 50.0 & 8.5 \\
\hline
\end{tabular}

CON, control diet, MLP, maternal low-protein diet, NAC, $N$-acetylcysteine.

${ }^{*}$ Two-way repeated measures ANOVA indicated that basal LVDP function following pre-treatment with NAC was influenced by sex $(P=0.04)$ and the interaction of sex, maternal diet and postnatal treatment $(P<0.01)$. Recovery over the first 30 min post-ischaemia was influenced by maternal diet $(P=0.003)$ and the interaction of sex and postnatal treatment $(P<0.05)$.

† Mean value was significantly different from that of the CON animals of the same sex $(P<0.01)$.

$\ddagger$ Mean value was close to being significantly different from that of the CON animals of the same sex $(P<0.055)$.

compared with baseline during both the early (recovery 1) and late (recovery 2) reperfusion phase (Table 2). However, the early reperfusion of the male heart (recovery 1) after saline treatment was significantly affected by maternal diet $(P<0 \cdot 003$; Fig. 1 (a)), with MLP-fed offspring exhibiting a blunted recovery from the challenge. For the later reperfusion response (recovery 2) there appeared a continued blunting of the response in MLP offspring; however, this just failed to achieve statistical significance $(P=0.055)$. In contrast, the early (recovery 1 ) and late (recovery 2 ) responses to reperfusion of hearts from females were similar between those exposed to the CON and low-protein diet in utero
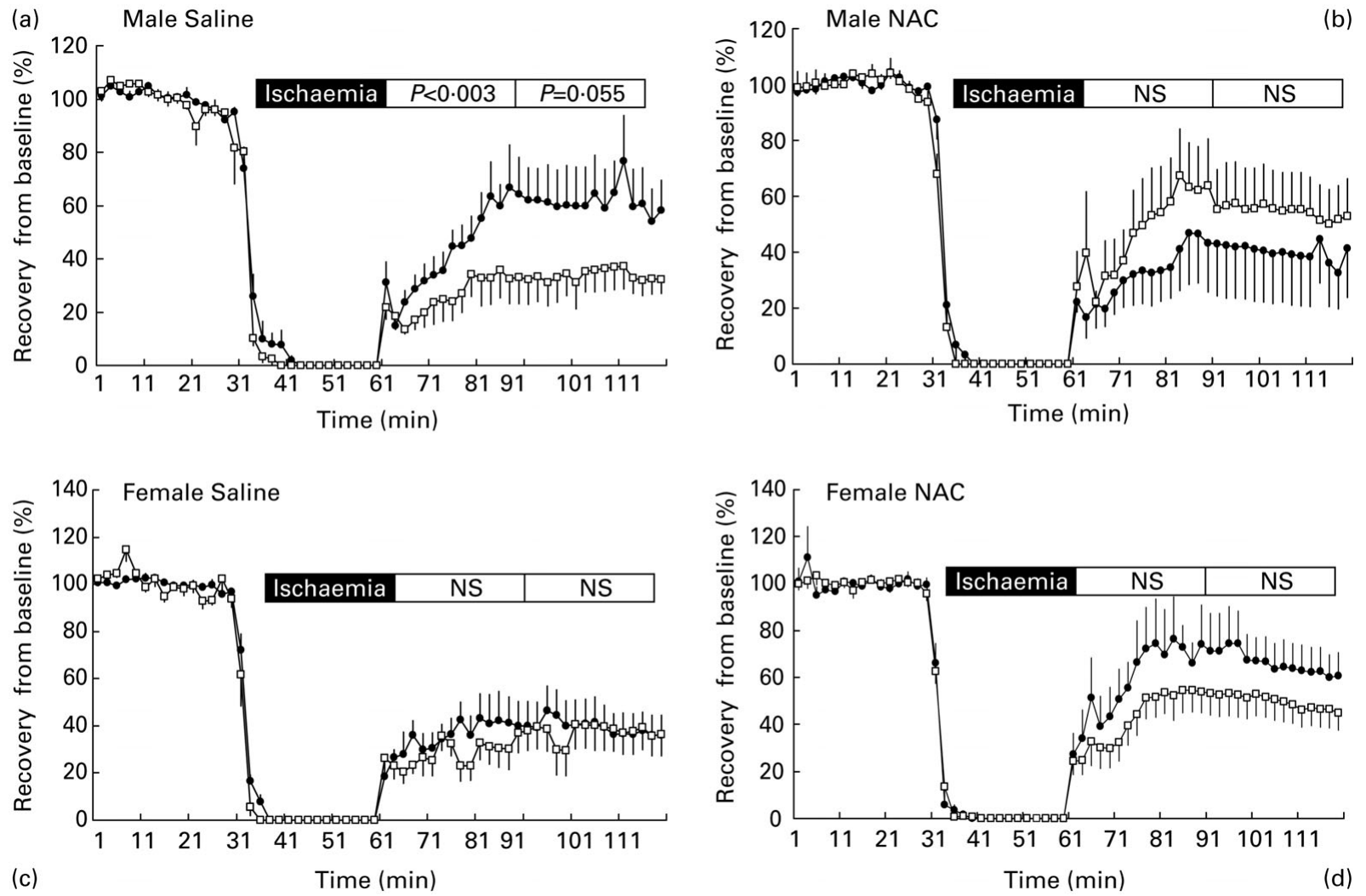

Fig. 1. Left ventricular function following ischaemia-reperfusion. Effects of pre-natal protein restriction on the percentage recovery from baseline of left ventricular developed pressure during 60 min post-ischaemic reperfusion in hearts from males treated with (a) saline (control (-๑-) $n$ 7; maternal-low protein diet (MLP; - $\square$ $n 7$ ) or (b) $N$-acetylcysteine (control $n 5$; MLP $n$ 11), and females treated with (c) saline (control $n 7$; MLP $n$ 6) or (d) $N$-acetylcysteine (control $n 7$; MLP $n$ 9). Values are means, with their standard errors represented by vertical bars. NS, not significantly different between dietary groups. 
(Fig. 1 (c)). Pre-treatment for $2 \mathrm{~d}$ of all offspring with NAC elicited a marked improvement on recovery of LVDP after $30 \mathrm{~min}$ ischaemia in all females irrespective of prenatal diet (Fig. 1 (d)) and in MLP males (Fig. 1 (b)). Importantly, NAC treatment prevented the blunting of the early recovery in male MLP offspring. However, in addition, there was a significant interaction between sex and treatment on the percentage recovery of LVDP $(P<0 \cdot 05)$. In this instance, NAC treatment was detrimental to the recovery of LVDP in CON males only (Fig. 1 (b)).

\section{Myocardial infarction}

Exposure to the MLP had no effect on myocardial infarct size after $30 \mathrm{~min}$ IR (Fig. 2). However, NAC treatment, of itself, significantly reduced myocardial infarct size experienced after $30 \mathrm{~min}$ ischaemia $(P<0 \cdot 001)$, but there was no effect of dietary treatment on this response.

\section{Discussion}

The present study is the first to show that male rats exposed to a low-protein diet throughout gestation suffer impaired recovery during reperfusion following myocardial ischaemia. This effect in the male 6-month-old offspring of MLP dams was prevented by $2 \mathrm{~d}$ pre-treatment with NAC - a potent free radical scavenger. In the offspring of protein-deprived rat dams, therefore, the response of the heart to ischaemia may be programmed by prenatal diet and may underpin myocardial recovery from mild-moderate coronary events. Cardiac function in female animals was largely unaffected by maternal protein restriction.

In keeping with our early studies, the offspring from rat dams fed a low-protein diet during pregnancy exhibited hypertension (Langley-Evans et al. 1996b; Gardner et al. 1997). The effects on blood pressure were not sex specific, as elevated systolic blood pressure was seen in both male and

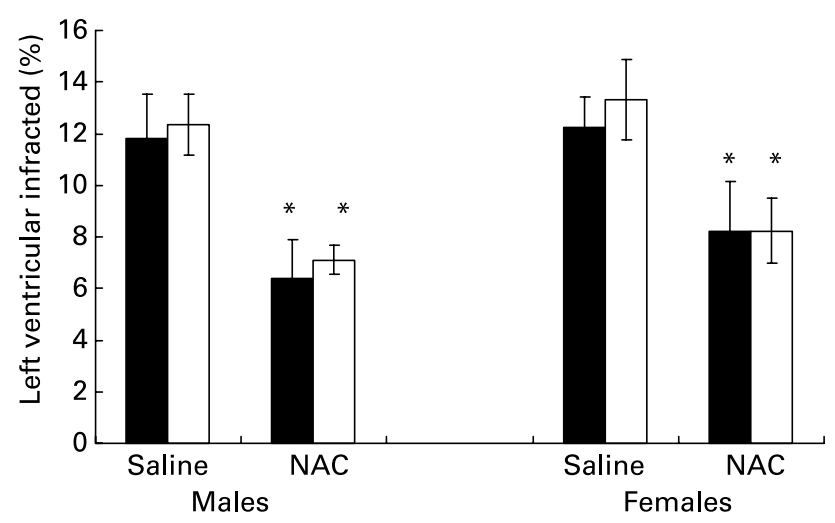

Fig. 2. Myocardial infarction. Effects of maternal low-protein diet ( $\square$ ) and $\mathrm{N}$-acetylcysteine (NAC) pre-treatment on left ventricular infarct size following $60 \mathrm{~min}$ reperfusion in the adult rat heart. Values are means, with their standard errors represented by vertical bars. * Mean value for NAC-treated rats was significantly lower than that for saline-treated rats $(P<0.001)$. ( $\square)$, Control animals. female offspring at 4 and 8 weeks of age. On occasion, other commonly used rat models of nutritionally induced hypertension have shown sex differences in the rate of rise in blood pressure with time (Gong et al. 1994), i.e. hypertension develops more rapidly and severely in male $v$. female offspring (Ozaki et al. 2001; Woods, 2004). Variation between studies is likely to be explained by differences in the macroand micronutrient composition of food given to the pregnant rats.

The elevation in blood pressure in MLP offspring at 4 weeks of age was not associated with an equivalent increase in heart rate which was similar between dietary groups. At 8 weeks of age, however, heart rate was significantly higher in MLP animals. Similarities in heart rate at 4 weeks of age have previously been shown by McMullen et al. (2004) but, unlike the present study, assessment was not carried out at a later age. The increased heart rate in MLP rats at 8 but not at 4 weeks of age may indicate maturation of cardiac $\beta$ adrenergic receptors in this group and increased tonic support from greater sympathetic innervation during blood pressure measurement at the later age, suggesting prenatal nutritional programming of autonomic nervous system activity. Nutritional programming of hypertension has been shown by Langley-Evans \& Jackson (1995) to persist for at least 10 months of age, and although a causal association between increased blood pressure and cardiac dysfunction in the present study is somewhat speculative, the available data suggest the assumption may be reasonable.

Using the established Langendorff technique to determine LVDP as a measure of basal cardiac function, we noted significant effects of prenatal diet, sex of the offspring and NAC treatment. Basal LVDP function, a measure of cardiac contractility, was similar in CON and MLP animals of the same sex. After pre-treatment with NAC, however, basal contractile function improved only in CON males and MLP females. As expected, global coronary ischaemia for $30 \mathrm{~min}$ followed by $60 \mathrm{~min}$ reperfusion significantly reduced LVDP in all offspring. However, of great interest was that in male but not female MLP offspring, the recovery of LVDP following ischaemia was significantly impaired by exposure to a maternal low-protein diet. The present study therefore shows for the first time that in the paradigm of nutritionally induced hypertension, low-protein-exposed rats show delayed recovery to myocardial ischaemia. This is a significant and common end-stage functional outcome of hypertension in the human population. In common with data in the human population, this effect was more pronounced in male, as opposed to female, offspring. Sex-specific effects are seen in other studies investigating CVD. Kahn et al. (2003) have provided evidence that dietary supplementation of lard during pregnancy in the rat leads to hypertension in the offspring but only in the females. Mechanisms leading to programmed hypertension and renal function in the lowprotein rat model also exhibit sex differences. The renal renin-angiotensin system contributes to the programming of hypertension but does so in a sex-specific, glucocorticoiddependent or -independent manner (McMullen \& LangleyEvans, 2005). In keeping with the present results, myocardial responses to ischaemia and reperfusion injury are known to differ between sexes. Female cardiomyocytes are more resistant to IR injury (Ranki et al. 2001) and the female 
heart exhibits reduced myocardial infarct size under experimental conditions (Wang et al. 2005).

The cellular mechanism for cardiac dysfunction and myocardial damage following IR injury is not fully established. Current evidence suggests that a burst of reactive oxygen species, rapidly generated upon reperfusion of tissues, plays a key role (Kaplan et al. 1997). NAC has been shown to act as a potent free radical scavenger and has been used as an effective therapeutic agent for heart disease (Arstall et al. 1995), given its ability to normalise left ventricular function following myocardial infarction (Sochman \& Peregrin, 1992). Indeed, in the present study pre-treatment with NAC clearly improved the recovery of cardiac function after $30 \mathrm{~min}$ IR in all MLP offspring and in CON females. Surprisingly, it appeared that pre-treatment with NAC in CON males was detrimental to post-ischaemic recovery in contractile function. The mechanism for this effect is unknown at present and needs further clarification in future studies. In contrast, pre-treatment of females with NAC improved recovery on contractile function in offspring exposed to either maternal diet, but as $\mathrm{CON}$ animals benefited more from NAC treatment it suggests that MLP animals have less ongoing oxidative stress within the heart. As expected, NAC pre-treatment also lessened the degree of myocardial infarction after IR in all treated offspring, but, in this regard, there was no effect of maternal dietary treatment. In this well-established protein-restriction model it is not currently known whether NAC has any effect on blood pressure but research in spontaneously hypertensive (Cabassi et al. 2001) and L-arginine methyl ester-treated rats (Rauchova et al. 2005) have shown that acute administration of NAC has been shown to attenuate the development of hypertension. This therefore may be an alternative mechanism through which NAC exerts beneficial effects in hearts from MLP animals.

One contradiction in the present study that cannot be readily reconciled is the apparent detrimental effect NAC had on the recovery of LVDP in CON male rats. It is generally accepted that previous antioxidant treatment attenuates myocardial damage after IR, but there are studies where this does not occur (for a review, see Dhalla et al. 2000). For example, Klawitter et al. (2002) found no protective effect of NAC on post-ischaemic cardiac contractile function in male Sprague-Dawley rats whereas, in the rabbit, increased protection has been observed using exactly the same protocol as the present study (Ambrosio et al. 1987). Such contradictions in the effects of NAC could be explained by differences in experimental protocols, or other variables including animal species, ischaemia duration, administered dose, or the method of NAC administration.

The data from the present study illustrate that cardiac contractile dysfunction after IR injury does not correlate with the degree of resulting myocardial infarction. For example, in saline-treated MLP rats, impaired recovery of LVDP was not associated with any increase in myocardial infarction. Similarly, in CON males, NAC pre-treatment blunted recovery of LVDP after IR, but no greater cardiac injury was observed. It is entirely possible and not unexpected that differing mechanisms exist for the apparent dissociation between physiological function and pathological consequence; one that regulates the intrinsic function of the heart post-ischaemia that is independent of antioxidant status and another that precipitates myocardial damage that is dependent on free radical scavenger activity. These potential factors controlling cardiac function following IR are unclear at present, but may well involve changes to intrinsic cardiac autonomic sensitivity.

An alternative explanation for the different isolated heart responses in CON and MLP offspring may be programmed changes to the anatomical development of the heart after maternal protein restriction. A recent study by $\mathrm{Xu}$ et al. (2006) investigated the effects of intra-uterine growth retardation resulting from maternal hypoxia and nutrient restriction on cardiac remodelling and impaired recovery in cardiac function following IR. The significant findings of this study included remodelling of cardiac tissue of the left ventricle, specifically increased expression of structural proteins, $\alpha$ and $\beta$ myosin heavy chains and collagen I and II. These changes were associated with cardiac dysfunction and increased susceptibility to IR injury which were seen in all intra-uterine growth retardation offspring. Intra-uterine growth retardation as a result of chronic hypoxia or maternal protein restriction also increases cardiomyocyte apoptosis (Bae et al. 2003) and reduces cardiomyocyte number at birth (Corstius et al. 2005). Lower numbers of ventricular myocytes are also noted in the stroke-prone spontaneously hypertensive rat compared with normotensive controls (van der Laarse et al. 1987). Cardiac myocyte differentiation is largely completed by term in the rat and subsequent postnatal cardiac growth is predominantly through individual myocyte hypertrophy, thus it is expected that reduced myocyte number at birth will persist into adulthood. In the protein-deprived rat heart, therefore, excessive hypertrophy may precipitate increased susceptibility to cardiac dysfunction in later life. Such a mechanism has been proposed to underpin the increased susceptibility to contractile dysfunction after IR in offspring that have been exposed to chronic hypoxia during late gestation (Li et al. 2003), and may well, in part, explain the results of the present study after exposure to an MLP.

In conclusion, we have demonstrated for the first time that prenatal protein restriction impairs recovery of the adult rat heart in a sex-specific manner after global coronary ischaemia, and that pre-treatment with the free radical scavenger NAC prevents this blunted recovery and significantly decreases myocardial infarct size. Prenatal protein restriction may contribute to the increased occurrence of CHD in low-birth-weight babies, or those that have suffered poor intra-uterine conditions. Poor recovery of contractile function did not, however, relate to myocardial infarct size and we therefore suggest that the factors regulating contractile function of the heart and myocardial infarct size are different. Further work on the developmental maturation of the heart, or its intrinsic autonomic activity, are implicated to determine any potential role in the prenatal programming of cardiac function during IR.

\section{Acknowledgements}

The present study was funded by a project grant from the British Heart Foundation. The authors acknowledge the expert technical assistance of Mr Richard Plant and Miss Sarah Kirkland. 


\section{References}

Alberola A, Such L, Gil F, Zaragoza R \& Morcillo EJ (1991) Protective effect of $N$-acetylcysteine on ischaemia-induced myocardial damage in canine heart. Naunyn Schmeiderbergs Arch Pharmacol 343, 505-510.

Ambrosio G, Zweier JL, Jacobus WE, Westfeldt ML \& Flaherty JT (1987) Improvement of post-ischaemic myocardial function and metabolism induced by administration of deferoxamine at the time of reflow: the role of iron in the pathogenesis of reperfusion injury. Circulation 76, 906-915.

Arstall MA, Yang J, Stafford I, Betts WH \& Horowitz JD (1995) $N$-acetylcysteine in combination with nitroglycerin and streptokinase for the treatment of evolving acute myocardial infarction. Circulation 92, 2855-2862.

Aruoma OI, Halliwell B, Hoey BM \& Butler J (1989) The antioxidant action of $\mathrm{N}$-acetylcysteine: its reaction with hydrogen peroxide, hydroxyl radical, superoxide, and hypochlorous acid. Free Radic Biol Med 6, 593-597.

Bae S, Xiao Y, Li G, Casiano CA \& Zhang L (2003) Effect of maternal chronic hypoxic exposure during gestation on apoptosis in fetal rat heart. Am J Physiol 285, H983-H990.

Barker DJP (1995) The fetal and infant origins of disease. Eur J Clin Invest 25, 457-463.

Bellinger L, Lilley C \& Langley-Evans SC (2004) Pre-natal exposure to a maternal low-protein diet programmes a preference for high fat foods in the young adult rat. Br J Nutr 92, 513-520.

Bolli R, Jeroudi MO, Patel BS, DuBose CM, Lai EK, Roberts R \& McCay PB (1989) Direct evidence that oxygen-derived free radicals contribute to post ischemic myocardial dysfunction in the intact dog, PNAS 86, 4695-4699.

Cabassi A, Dumont EC, Girouard H, Bouchard JF, Le Jossec M, Lamontagne D, Besner JG \& de Champlain J (2001) Effects of chronic $\mathrm{N}$-acetylcysteine treatment on the actions of peroxynitrite on aortic vascular reactivity in hypertensive rats. J Hypertens 19, 1233-1244.

Corstius HB, Zimanyi MA, Maka N, Herath T, Thomas W, van der Laarse A, Wreford NG \& Black MJ (2005) Effect of intrauterine growth restriction on the number of cardiomyocytes in rat hearts. Pediatr Res 57, 796-800.

Dhalla NS, Elmoselhi AB, Hata T \& Makino N (2000) Status of myocardial antioxidants in ischemia-reperfusion injury. Cardiovas Res 47, 446-456.

Fall CH, Vijayakumar M, Barker DJP, Osmond C \& Duggleby S (1995) Weight in infancy and prevalence of coronary heart disease in adult life. BMJ 310, 17-19.

Festing MF (2006) Design and statistical methods in studies using animal models of development. ILAR J 47, 5-14.

Gardner DS, Jackson AA \& Langley-Evans SC (1997) Maintenance of maternal diet-induced hypertension in the rat is dependent upon glucocorticoids. Hypertension 30, 1525-1530.

Gong G, Dobin A, McArdle S, Sun L, Johnson ML \& Pettinger WA (1994) Sex influence on renal $\alpha 2$-adrenergic receptor density in the spontaneously hypertensive rat. Hypertension 23, 607-612.

Great Britain Home Office (2000) Home Office Guidance on the Operation of the Animals (Scientific Procedures) Act 1986. London: The Stationery Office.

Han HC, Austin KJ, Nathanielsz PW, Ford SP, Nijland MJ \& Hanson TR (2004) Maternal nutrient restriction alters gene expression in the ovine fetal heart. $J$ Physiol 558, 111-121.

Kahn IY, Taylor PD, Dekou V, Seed PT, Lakasing L, Graham D, Dominiczak AF, Hanson MA \& Poston L (2003) Gender linked hypertension in offspring of lard fed pregnants rats. Hypertension 41, 168-175.

Kaplan P, Lehotsky J \& Rakan P (1997) Role of sarcoplasmic reticulum in the contractile dysfunction during myocardial ischaemia and reperfusion. Physiol Res 46, 333-339.
Klawitter PF, Murray HN, Clanton TL \& Angelos MG (2002) Reactive oxygen species generated during myocardial ischemia enable energetic recovery during reperfusion. Am $J$ Physiol 283, H1656-H1661.

Kwong WY, Wild AE, Roberts P, Willis AC \& Fleming TP (2000) Maternal undernutrition during preimplantation period of rat development causes blastocyst abnormalities and programming of post natal hypertension. Development 127, 4195-4202.

Langley SC \& Jackson AA (1994) Increased systolic blood pressure in adult rats induced by fetal exposure to maternal low protein diet. Clin Sci 86, 217-222.

Langley SC \& Kelly FJ (1992) Effect of food restriction on hyperoxia-induced lung injury in pre-term guinea pig. Am $J$ Physiol 263, L357-L362.

Langley-Evans SC (2006) Developmental programming of health and disease. Proc Nutr Soc 65, 97-105.

Langley-Evans SC, Gardner DS \& Jackson AA (1996a) Maternal protein restriction influences the programming of the rat hypothalamic-pituitary-adrenal axis. J Nutr 126, 1578-1585.

Langley-Evans SC \& Jackson AA (1995) Captopril normalises systolic blood pressure in rats with hypertension induced by fetal exposure to maternal low protein diets. Comp Biochem Physiol 110A, 223-228.

Langley-Evans SC, Phillips GJ \& Jackson AA (1994) In utero exposure to maternal low protein diets induces hypertension in weanling rats, independently of maternal blood pressure changes. Clin Nutr 13, 319-324.

Langley-Evans SC, Welham SJM \& Jackson AA (1999) Fetal exposure to maternal low protein diets impairs nephrogenesis and promotes hypertension in the rat. Life Sci 64, 965-974.

Langley-Evans SC, Welham SJM, Sherman JC \& Jackson AA (1996b) Weanling rats exposed to maternal low protein diets during discrete periods of gestation exhibit differing severity of hypertension. Clin Sci 91, 607-615.

Law CM, de Swiet M \& Osmond C (1993) Initiation of hypertension in-utero and its amplification throughout life. BMJ 306, 24-27.

Leipala JA, Boldt T, Turpeinen U, Vuolteenaho O \& Fellman V (2003) Cardiac hypertrophy and altered hemodynamic adaptation in growth-restricted pre-term infants. Pediatr Res 53, 989-993.

Li G, Xiao Y, Estrell JL, Ducsay CA, Gilbert RD \& Zhang L (2003) Effect of fetal hypoxia on heart susceptibility to ischemia and reperfusion injury in the adult rat. J Soc Gyneol Invest 10, 265-274.

McMullen S, Gardner DS \& Langley-Evans SC (2004) Prenatal programming of angiotensin II type 2 receptor expression in the rat. $\mathrm{Br}$ J Nutr 91, 133-140.

McMullen S \& Langley-Evans SC (2005) Maternal low-protein diet in rat pregnancy programs blood pressure through sex specific mechanisms. Am J Physiol 288, R85-R90.

Ozaki T, Nishina H, Hanson MA \& Poston L (2001) Dietary restriction in pregnant rats causes gender-related hypertension and vascular dysfunction in offspring. J Physiol 530, 141-152.

Peterson S \& Raynor M (2002) Coronary Heart Disease Statistics 10. Database (http://www.heartstats.org/publications). London: The British Heart Foundation.

Ranki HJ, Budas GR, Crawford RM \& Jovanovic A (2001) Genderspecific difference in cardiac ATP-sensitive $\mathrm{K}^{+}$channels. $J$ Am Coll Cardiol 38, 906-915.

Rauchova H, Pechanova O, Kunes J, Vokurkova M, Dobesova Z \& Zicha $\mathrm{J}$ (2005) Chronic $N$-acetylcysteine administration prevents the development of hypertension in N $(\omega)$-nitro-L-arginine methyl ester-treated rats: the role of reactive oxygen species. Hypertens Res 28, 475-482.

Sherman RC \& Langley-Evans SC (2000) Antihypertensive treatment in early postnatal life modulates prenatal dietary influences upon blood pressure in the rat. Clin Sci 98, 269-275.

Sochman J, Kolc J, Vrana M \& Fabian J (1990) Cardioprotective effects of $\mathrm{N}$-acetylcysteine: the reduction in the extent of infarction and occurrence of reperfusion arrhythmias in the dog. Int J Cardiol 28, 191-196. 
Sochman J \& Peregrin JH (1992) Total recovery of left ventricular function after acute myocardial infarction: comprehensive therapy with streptokinase, $N$-acetylcysteine and percutaneous transluminal coronary angioplasty. Int J Cardiol 35, 116-118

van der Laarse A, Bloys van Treslong CH, Vliegen HW \& Ricciardi L (1987) Relation between ventricular DNA content and number of myocytes in hearts of normotensive and spontaneously hypertensive rats. Cardiovasc Res 21, 223-229.

Veille JC, Hanson R, Sivakoff M, Hoen H \& Ben-Anui M (1993) Fetal cardiac size in normal intrauterine growth retarded and diabetic pregnancies. Am J Perinat 10, 275-279.

Wang M, Baker L, Tsai BM, Meldrum KK \& Meldrum DR (2005) Sex specific differences in the myocardial inflammatory response to ischaemia/reperfusion injury. Am J Physiol 285, H687-H692.

Williams RE, Zweier JL \& Flaherty JT (1991) Treatment with deferoxamine during ischaemia improves functional and metabolic recovery and reduces reperfusion-induced oxygen radical generation in rabbit hearts. Circulation 83, 1006-1014.

Woods LL (2004) Renal disease and fetal undernutrition. In Fetal Nutrition and Adult Disease: Programming of Chronic Disease through Fetal Exposure to Undernutrition, pp. 235-258 [SC Langley-Evans, editor]. Wallingford, UK: CABI.

Xu Y, Williams SJ, O'Brien D \& Davidge ST (2006) Hypoxia or nutrient restriction during pregnancy in rats leads to progressive cardiac remodelling and impairs postischaemic recovery in adult male offspring. FASEB $J \mathbf{2 0}$, E536-E545. 